\title{
A Vertical Handover Management for Mobile Telemedicine System using Heterogeneous Wireless Networks
}

\author{
Hoe-Tung Yew \\ School of Engineering, \\ Universiti Malaysia Sabah, \\ Sabah, \\ Malaysia
}

\begin{abstract}
Application of existing mobile telemedicine system is restricted by the imperfection of network coverage, network capacity, and mobility. In this paper, a novel telemedicine based handover decision making (THODM) algorithm is proposed for mobile telemedicine system using heterogeneous wireless networks. The proposed algorithm select the best network based on the services requirement to ensure the connected or targeted network candidate has sufficient capacity for supporting the telemedicine services. The simulation results show that the proposed algorithm minimizes the number of unnecessary handover to WLAN in high speed environment. The throughput achieved by the proposed algorithm is up to $75 \%$ and $205 \%$ higher than Cellular and RSS based schemes, respectively. Moreover, the average data transmission cost of THODM algorithm is $\mathbf{2 4 \%}$ and $\mathbf{6 9 . 2 \%}$ lower than the Cellular and RSS schemes. The proposed algorithm minimizes the average transmission cost while maintaining the telemedicine service quality at the highest level in high speed environment.
\end{abstract}

Keywords-Mobile telemedicine system; vertical handover; heterogeneous networks; unnecessary handover; throughput; cost

\section{INTRODUCTION}

The rapid growth of wireless communication technologies has led to the development of telemedicine. Telemedicine provides remote monitoring and diagnosis services via information and communication technologies. Wireless Local Area Network (WLAN), Worldwide Interoperability for Microwave Access (WiMAX) and cellular networks are the three wireless technologies widely applied in telemedicine.

WLAN is the most preferable by users due to the high transmission capacity and low network access cost, however, the small coverage area limits the user's mobility support. WLAN based telemedicine systems are typically for indoor application (home, hospital, clinic, etc.) [1-4]. To tackle the issue in WLAN, researches on cellular network based telemedicine were raised. Authors in [5-8] present a cellular network based telemedicine system. The advantages of cellular based telemedicine system are that it supports high mobility and offers large service coverage. However, the capacity of cellular network is insufficient for high quality images and continuous video transmission as the channel bandwidth is limited. The high bandwidth Fourth Generation Long Term Evolution (4G-LTE) system is still under

This research was supported by Ministry of Education Malaysia and Universiti Teknologi Malaysia, Grant Q.J130000.2745.02K17.

\author{
Eko Supriyanto, M Haikal Satria* and Yuan-Wen Hau \\ IJN-UTM Cardiovascular Engineering Centre \\ Faculty of Biosciences and Medical Engineering, \\ Universiti Teknologi Malaysia \\ Johor, Malaysia
}

deployment. The coverage is imperfect in rural and suburban areas. Consequently, the use of 4G-LTE in telemedicine is limited.

The performance of telemedicine service is dependent on the network quality. Poor network quality will disrupt the health data in transmission. Authors in $[9,10]$ proposed WiMAX based telemedicine application to provide higher bandwidth than cellular network with extended network coverage than WLAN. Authors in [11] integrate both WLAN and WiMAX networks where WLAN is for indoor application and WiMAX is for outdoor environment. However, handover scheme between WLAN and WiMAX is not discussed by authors. WiMAX technology overcomes the issues of small coverage and insufficient bandwidth encountered by WLAN and cellular network, respectively. Unfortunately, most of the network service providers are ceasing development of WiMAX [12]. As a result, the mobile telemedicine system that relies on WiMAX technology cannot guarantee the users connect continuously to the telemedicine services provider at anywhere due to the imperfection of network coverage.

Each wireless technology has its own advantages and disadvantages. None of them can fully support the telemedicine services in terms of data transmission rate and mobility. Therefore, application of existing mobile telemedicine system is sometimes restricted by mobility, coverage and constraints of data transmission rate issues. The continuous service connection and guarantee of data transmission rate are the two main factors to maintain the quality of telemedicine services. For this purpose, a mobile telemedicine system that has capability of accessing multiple wireless networks is needed so that the system has wider service coverage and guarantee the service quality by connecting to the best network based on the services requirement.

The rest of the paper is organized as follow. Section II reviews the existing handover algorithms in heterogeneous networks. In Section III, the framework of mobile telemedicine is introduced. Section IV descries the proposed THODM handover algorithm for mobile telemedicine system. The performance of the proposed algorithm is discussed in Section V. Finally, Section VI concludes the paper. 


\section{VERTICAL HANDOVER IN HETEROGENEOUS NETWORKS}

Vertical handover in heterogeneous network is a process of the mobile terminal (MT) migrating network connection from one network technology to another. The vertical handover process can be divided into three phases which are handover initiation, decision and execution [13]. The handover initiation phase discovers and obtains available network information via Media Independent Handover Function (MIHF) [14]. The handover decision is a process of selecting the most suitable network based on the calculated network information and triggering handover at the right time. Executive phase is establishing the connection with targeted network and releasing the old network.

Numerous handover decision making algorithms have been previously proposed. A received signal strength (RSS) based handover algorithm introduced by $[15,16]$ reduces handover failure rate from WLAN to cellular network based on the MT's speed and handover latency. Authors in [17, 18] proposed a prediction based handover decision scheme to estimate MT dwelling time in WLAN coverage. Mobile terminal triggers handover to WLAN cell if and only if the estimated dwelling time is greater than the time threshold. However, high handover delay is observed because these methods need to take two RSS sample points $\left(\mathrm{P}_{1}\right.$ and $\mathrm{P}_{2}$ in Fig. 1) within WLAN coverage for dwelling time estimation process. This processing time will reduce the dwelling time within WLAN as soon as the handover process is done at $\mathrm{P}_{2}$.

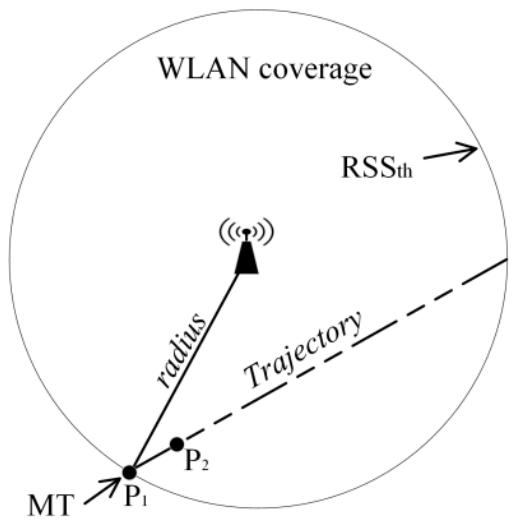

Fig. 1. Scenario of prediction process in $[17,18]$

Cost function based handover algorithm for wireless wide area network (WWAN) and WLAN integrated networks has been proposed by [19]. The results showed that WLAN is more preferred than WWAN due to the low network access cost. Also, authors in [20] proposed a cost per signal-to-noiseplus-interference-ratio (SINR) function to improve the throughput and reduce the cost for accessing the integrated wireless networks (WLAN and WWAN). In this approach, authors assumed total cost equal to packet transmission cost plus handover processing cost. However, MT's velocity is not considered by these schemes. The small coverage of WLAN cell will lead to high number of unnecessary handovers in high mobility.
Authors in [21-23] proposed a multiple attribute decision making (MADM) based handover algorithms. In these schemes, a weighting system is given to the handover criteria based on user preference. The network candidate that scores the highest weight sum is selected as a handover target. Recently, intelligent based MADM handover algorithm is presented to improve the performance of handover. A NeuroFuzzy based MADM handover algorithm is proposed by [24]. Authors in [25] presented Fuzzy based MADM handover algorithm. Furthermore, Artificial Neural Network (ANN) based handover decision making in heterogeneous networks is presented in [26]. The disadvantage of using intelligent based handover algorithm is high handover latency caused by ANN learning/training, and Fuzzy Logic fuzzification or defuzzification processes. Moreover, handover latency further increases while more handover criteria are taken into account.

In high speed environment, most of the handover algorithms optimize their performance by predefining a speed threshold for WLAN. MT triggers handover to WLAN if and only if MT's speed is below the predefined speed threshold to avoid the handover failure and unnecessary handover to WLAN. The application of WLAN is restricted to static or pedestrian navigation environment [27]. For example, authors in $[21,28-30]$ predefined the speed threshold for WLAN at $10 \mathrm{~m} / \mathrm{s}$ and below (depending on the radius of WLAN). In addition, Fuzzy MADM based handover algorithm presented by [25] and [31] set the fuzzy if-else rule, "if MT velocity is low then the probability of rejecting WLAN is low; else the probability of rejecting WLAN is high". In this paper, Telemedicine based vertical handover decision making (THODM) algorithm is proposed for mobile telemedicine system aims to maintain the quality of telemedicine service at the highest level with minimum cost in high speed environment.

\section{FRAMEWORK OF MOBILE TELEMEDICINE SYSTEM IN HETEROGNEOUS NETWORKS}

Fig. 2 shows the proposed mobile telemedicine system framework. The telemedicine device is integrated with various type of electronic health sensors such as pulse oximetry, body temperature and Electrocardiogram (ECG) sensors. The signals or data collected by these sensors will be analyzed by an embedded self-interpretation algorithm [7]. In case an abnormal health signal is detected, the system will set patient health condition (PHC) to "LOW" or " 0 " and give priority to "Critical" buffer to transmit the abnormal health signal to hospital to let the patient get treatment promptly. The PHC is set to "HIGH" or " 1 " when the patient is in normal health condition. The health data is stored in the "Non-critical" buffer queue for transmission via WLAN or cellular network.

Telemedicine based handover decision making (THODM) algorithm assists the device to select the best wireless network to transmit the patient's health data to hospital based on the inputs from accelerometer, MIHF, user setting (e.g. video, audio, signal, image, etc.) and predefined database. The function of these modules is illustrated in TABLE I. 


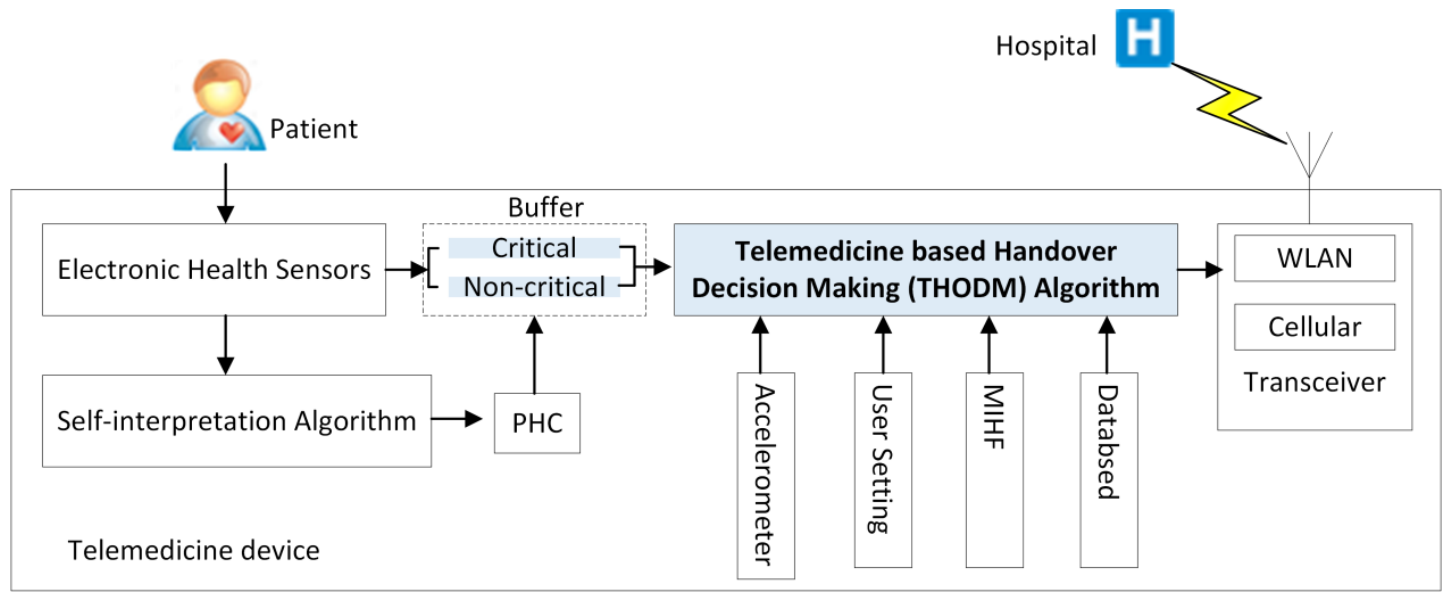

Fig. 2. Mobile Telemedicine Framework

TABLE I. INPUT MODULES OF THODM ALGORITHM

\begin{tabular}{ll}
\hline Modules & Function \\
\hline Accelerometer & Measure the MT traveling speed. \\
MIHF & $\begin{array}{l}\text { Discover neighbouring networks and measure the } \\
\text { network quality. }\end{array}$ \\
User setting & $\begin{array}{l}\text { Monitor type of telemedicine services applied by user. } \\
\text { Contains predefined values such as RSS threshold, } \\
\text { network tariff rate, and minimum data rate required by } \\
\text { each type of telemedicine service. These values will } \\
\text { be the reference values for THODM algorithm during } \\
\text { handover decision making. }\end{array}$ \\
\hline
\end{tabular}

\section{THODM ALGORITHM}

THODM algorithm consists three structured phases of self-inspection, pre-handover filtering and network selection. The handover parameters required by this algorithm are RSS, Signal-to-Noise ratio $(S N R)$, data rate requirement $\left(D R_{R E Q}\right)$, cost $(C)$, and velocity $(v)$. The RSS and SNR parameters can be obtained via MIHF. The MT's velocity can be measured using an accelerometer. The network access cost and $D R_{R E Q}$ are predefined and stored in the database or memory of the telemedicine device.

RSS measurement is used to discover the neighboring wireless networks. The SNR parameter is for algorithm to evaluate the capacity of the available networks. In order to guarantee the quality of telemedicine services, the connected or targeted network candidate must has sufficient capacity to support the data rate required by telemedicine services. The priority level and $D R_{R E Q}$ of telemedicine services is shown in Table II.

THODM algorithm also takes network tariff rate into consideration with the purpose of reducing the data transmission cost of telemedicine services. The parameter velocity is used to estimate the dwelling time within WLAN coverage. The energy saving issue is not considered in this work because THODM algorithm is mainly designed for high speed environment such as ambulance or vehicle based telemedicine system. The system could be powered by the power source from ambulance or vehicle.
TABLE II. DATA RATE REQUIRED By DIFFERENT TYPE OF TELEMEDICINE SERVICES $[32,33]$

\begin{tabular}{|c|c|c|c|}
\hline Data type & Telemedicine service & Data rate $\left(D R_{R E Q}\right)$ & $\begin{array}{l}\text { Priority } \\
\text { Level }\end{array}$ \\
\hline \multirow{3}{*}{$\begin{array}{l}\text { Biosignal } \\
\text { data }\end{array}$} & ECG (12 channels) & $24 \mathrm{kbps}$ & \multirow{3}{*}{1} \\
\hline & Heart rate & $2-5 \mathrm{kbps}$ & \\
\hline & Blood pressure & $2-5 \mathrm{kbps}$ & \\
\hline Audio & Voice & $4-25 \mathrm{kbps}$ & 2 \\
\hline \multirow[b]{2}{*}{ File transfer } & Uncompressed image & 30-40Mbytes & \multirow[b]{2}{*}{3} \\
\hline & $\begin{array}{l}\text { Region-of-interest } \\
\text { (ROI) JPEG image }\end{array}$ & 15-19Mbytes & \\
\hline Video & Diagnostic video & 768kbps-10Mbps & 4 \\
\hline
\end{tabular}

\section{A. Self-inspection Phase}

In self-inspection phase, THODM algorithm monitors the RSS of current connected network $\left(R S S_{C C N}\right)$, SNR of current connected network $\left(S N R_{C C N}\right)$, and $D R_{R E Q}$ periodically to ensure the capacity of current connected network fulfills the services requirements. The current connected network $(C C N)$ can be WLAN or cellular network. Assumed the cellular technology that integrated in the telemedicine device (Fig. 2) is Universal Mobile Telecommunication System (UMTS) network. The proposed algorithm is continuously searching for WLAN if the current connected network is not a WLAN. THODM algorithm gives priority to WLAN because WLAN provides high capacity with lower cost. The transmission cost can be reduced by optimizing the connection to WLAN. The quality of current connected network $\left(Q_{C C N}\right)$ is determined by:

$$
Q_{C C N}=F\left(R S S_{C C N}\right) *\left(S N R_{C C N}-S N R_{R E Q_{-} C C N}\right)
$$

where $F\left(R S S_{C C N}\right)$ is a unit step function as shown in (2) where the output is equal to 1 if $R S S_{C C N}$ is greater than $R S S_{C C N}$ threshold $\left(R S S_{t h C C N}\right)$, otherwise 0 .

$F(R S S)=F\left(R S S_{C C N}-R S S_{t h C C N}\right) \begin{cases}0, & R S S_{C C N} \leq R S S_{t h C C N} \\ 1, & R S S_{C C N}>R S S_{t h C C N}\end{cases}$

$S N R_{R E Q C C N}$ is a dynamic SNR threshold defined based on the sum of the data rate of the telemedicine services that 
applied by user, $D R_{R E Q}$. It can be calculated by using Shannon-Hartley theorem and given as

$$
\begin{gathered}
D R_{R E Q}=W_{C C N} \log _{2}\left(1+S N R_{R E Q_{-} C C N}\right) \\
S N R_{R E Q_{-} C C N}=2^{\frac{D R_{R E Q}}{W_{C C N}}}-1
\end{gathered}
$$

where $W_{C C N}$ is bandwidth of current connected network. Since SNR of the WLAN and cellular network cannot be compared directly, we standardize the SNR threshold $\left(S N R_{R E Q}\right)$ for both WLAN and UMTS networks by selecting $S N R_{R E Q}$ of WLAN $\left(S N R_{R E Q \_W L A N}\right)$ as a reference value. According to Shannon-Hartley theorem, the $D R_{R E Q}$ of WLAN and UMTS channel can be calculated by

$$
\begin{aligned}
& D R_{R E Q}=W_{W L A N} \log _{2}\left(1+S N R_{R E Q_{-} W L A N}\right) \\
& D R_{R E Q}=W_{U M T S} \log _{2}\left(1+S N R_{\text {REQ_UMTS }}\right)
\end{aligned}
$$

Where $S N R_{R E Q} U M T S$ is $S N R_{R E Q}$ of UMTS and $W_{W L A N}$ and $W_{U M T S}$ represent channel bandwidth $(\mathrm{Hz})$ of WLAN and UMTS networks, respectively. Assuming $D R_{R E Q}$ for both (4) and (5) are identical, we substitute (4) into (5). The relationship between $S N R_{R E Q_{-} W L A N}$ and $S N R_{R E Q} Q_{-} U M T S$ is given as:

$$
\begin{gathered}
\log _{2}\left(1+S N R_{R E Q \_W L A N}\right)=\frac{W_{U M T S}}{W_{W L A N}} \log _{2}\left(1+S N R_{R E Q_{-} U M T S}\right) \\
S N R_{R E Q_{\_} W L A N}=\left(1+S N R_{R E Q_{\_} U M T S}\right)^{\frac{W_{U M T S}}{W_{W L A N}}-1}
\end{gathered}
$$

By replacing $S N R_{R E Q}$ UMTS in (6) with measured UMTS SNR value ( $\left.S N R_{U M T S}\right)$, we can obtain an equivalent $S N R$ value in WLAN (E_SNR $\left.R_{U M T S}\right)$.

$$
E_{-} S N R_{U M T S}=\left(1+S N R_{U M T S}\right)^{\frac{W_{U M T S}}{W_{W L A N}}}-1
$$

Therefore, set of SNR values for both WLAN and UMTS networks is given by:

Rewrite (1),

$$
S N R=S N R_{W L A N} \cup E_{-} S N R_{U M T S}
$$

$$
Q_{C C N}=F\left(R S S_{C C N}\right) *\left(S N R-S N R_{R E Q_{-} W L A N}\right)
$$

In the case of $Q_{C C N} \leq 0$ or $C C N \neq$ WLAN, THODM algorithm scans for neighboring wireless network to find a better network candidate to support the telemedicine services. The detected available network candidates will proceed to the pre-handover filtering phase. Conversely, if no available network is detected, the proposed algorithm will deactivate the lowest priority service systematically in order to adapt to the current connect network [34]. The higher priority services that supportable by current connect network are remaining active with guarantee of service quality.

\section{B. Pre-Handover Filtering Phase}

Pre-handover filtering phase consists of dwelling time prediction and network quality evaluation processes. The dwelling time prediction process applies to WLAN cell only whereas the network quality evaluation process is applied to all the available networks. The aim of dwelling time prediction process is to avoid unnecessary handover to WLAN in high speed environment. The proposed dwelling time prediction process predefined two RSS thresholds: RSS boundary $\left(R S S_{\text {boundary }}\right)$ and RSS threshold $\left(R S S_{t h}\right) . R S S_{\text {boundary }}$ represents the edge of the WLAN coverage and $R S S_{t h}$ denotes minimum RSS for reliable packet delivery. MT initiates prediction process once the measured $\operatorname{RSS}\left(R S S_{W L A N}\right)$ is greater than $R S S_{\text {boundary }}$.

Fig. 3 shows the scenario of MT traveling within the WLAN coverage. MT enters the WLAN coverage at point $\mathrm{P}_{\text {entry }}$ and exits at point $\mathrm{P}_{\text {exit }} \cdot R$ is the radius of WLAN cell, $r$ is distance between $\mathrm{P}_{\text {In_RSSth }}$ and WLAN access point (AP), $l$ is distance between $\mathrm{P}_{\text {In_RSSth }}$ and $\mathrm{P}_{\text {Out_RSSth }}$, and $d$ denotes $\mathrm{MT}$

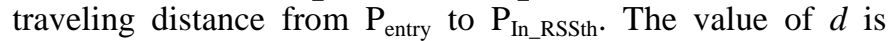
varying according to the MT's direction of motion from $\mathrm{P}_{\text {entry }}$.

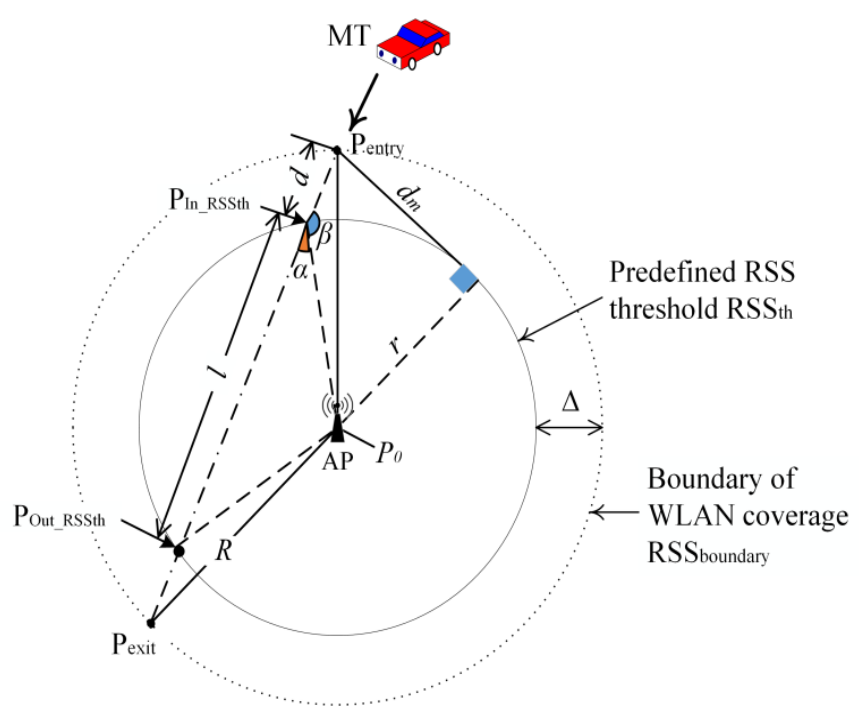

Fig. 3. MT trajectory in WLAN coverage

The distance $d$ is determined by

$$
d=t_{d} * v, \quad \Delta<d<d_{m}
$$

where $t_{d}$ is MT's traveling time from $\mathrm{P}_{\text {entry }}$ to $\mathrm{P}_{\text {In_RSSth }}$, and $v$ is MT's velocity. The $\Delta$ and $d_{m}$ are given as

$$
\begin{aligned}
\Delta & =R-r \\
d_{m} & =\sqrt{R^{2}-r^{2}}
\end{aligned}
$$

The $R$ and $r$ values can be calculated by using the Logdistance path loss model [35], expressed as

$$
\begin{gathered}
\overline{R S S}_{\text {boundary }}=P_{T X}-P L_{0}-10 n \log \frac{R}{d_{0}}+\varepsilon \\
R=d_{0} * 10\left(\frac{P_{T X}-P L_{0}-\overline{R S S}_{\text {boundary }}+\varepsilon}{10 n}\right)
\end{gathered}
$$

where $P_{T X}$ is AP transmit power, $d_{0}$ is the distance from AP to reference point $\left(P_{0}\right)$ usually $1 \mathrm{~m}, P L_{0}$ is the power loss at $P_{0}, \overline{R S S}_{\text {boundary }}$ is mean of $R S S_{\text {boundary }}, n$ is path loss exponent, and $\varepsilon$ is a zero-mean Gaussian random variable caused by shadow fading. Similarly, the $r$ value can be measured by replacing $\overline{R S S}_{\text {boundary }}$ in (13) with $\overline{R S S}_{t h}$. The $\overline{R S S}_{\text {boundary }}$ can be calculated by

$$
\overline{R S S}_{\text {boundary }}=\frac{1}{N} \sum_{i=0}^{i=N} R S S_{i}
$$

where $N$ is number of samples. $N$ is adjusted dynamically to the MT's velocity. It given as

$$
N=\left\lceil\varphi * \frac{L}{v * T_{S}}\right\rceil \quad \varphi \in\{0.1,0.2, \ldots, 0.9\}
$$


where $T_{s}$ is RSS sampling time (5 ms [36]) and $L$ is a fixed distance of $1 \mathrm{~m}$ [37]. In this work, MT monitors $R S S_{W L A N}$ periodically in every meter. The higher the MT's velocity is, the smaller the sample size. The maximum $N$ is limited to 20 to prevent over sampling when MT is at low mobility. The impact of Doppler shift can be mitigated by using the Doppler frequency offset estimation and compensation algorithms that presented in [38, 39].

The distance $l$ can be determined by using a trigonometric function as follows:

$$
\begin{aligned}
& \cos \alpha=\frac{l}{2 r} \\
& l=2 r \cos \alpha,
\end{aligned}
$$

where $\alpha=\pi-\beta$ (Fig. 3) and $r$ can be calculated by using (13). Rewrite (16),

$$
l=-2 r \cos \beta
$$

By using Law of cosine, angle $\beta$ can be calculated by

$$
\begin{aligned}
R^{2} & =r^{2}+d^{2}-2 r d \cos \beta \\
\beta & =\cos ^{-1}\left(-\frac{R^{2}-r^{2}-d^{2}}{2 d r}\right)
\end{aligned}
$$

Substitute (18) into (17), estimated traveling distance $l$ is given as

$$
l=\frac{R^{2}-r^{2}-d^{2}}{d}
$$

The estimated beneficial time to MT within the WLAN coverage $\left(T_{W L A N}\right)$ can be determined by

$$
\begin{gathered}
T_{W L A N}=\frac{l}{v} \\
T_{W L A N}=\frac{R^{2}-r^{2}-d^{2}}{d v}
\end{gathered}
$$

The duration of $T_{W L A N}$ is depending on the $R, r, d$ and $v$. The WLAN cell which estimated $T_{W L A N}$ is greater than the threshold time $\left(T_{W L A N_{-} t h}\right)$ proceeds to network quality evaluation process, otherwise rejected. The $T_{W L A N_{-} t h}$ for unnecessary handover is 2 seconds [17, 18]. The dwelling time in proposed method will be predicted as soon as MT detected $R S S_{t h}$. This improves the previous method in $[17,18]$, where the dwelling time prediction is initiated after MT detected $R S S_{t h}$. By using two predefined thresholds, the proposed method reduces the prediction processing time within the dwelling time.

The network quality evaluation process evaluates the quality of all available network candidates except the WLAN cell which estimated $T_{W L A N}$ is less than $T_{W L A N_{-} t h}$. The quality of each network candidate $(Q)$ is evaluated based on the measured $R S S, S N R$ and $C$ values. $Q$ is given as

$$
Q_{k}=\frac{F\left(R S S_{k}\right)\left(S N R-S N R_{R E Q} \_W L A N\right)}{C_{k}}, k=\{1,2, \ldots n\}
$$

where each network candidate is represented by $k$ of $n$ candidates and $C_{k}$ is predefined cost per $\mathrm{Mb}$ of network candidate $k$.

In this process, the network candidate which scores less than or equal to zero $\left(Q_{k} \leq 0\right)$ will be rejected. Only the qualify network candidate that is greater than zero $\left(Q_{k}>0\right)$ will be added to a qualify network list (QNL) for network selection phase.

\section{Network Selection Phase}

The network selection phase usually falls into three possible conditions. The first condition is that the number of network candidates in QNL $\left(U_{Q N L}\right)$ is equal to zero. In such case, MT will adjust the services requirement $D R_{R E Q}$ by removing the lowest priority service and back to the selfinspection phase. Next condition is only one network candidate in QNL $\left(U_{Q N L}=1\right)$. MT triggers handover to the sole network directly. The last condition is that $U_{Q N L}>1$. Typically, the network candidate which has the highest score will be selected as a handover target or the best network. The best network $(\mathscr{B})$ is given as

$$
\mathscr{B}=\max \left(Q_{k}\right)
$$

\section{RESULTS AND DISCUSSION}

In this section, performance of the THODM algorithm is evaluated by its number of unnecessary handovers, throughput, and cost of transmission. The evaluation is done by simulating the proposed algorithm at high speed environment $(50 \mathrm{~km} / \mathrm{h}$ to $120 \mathrm{~km} / \mathrm{h})$. Fig. 4 shows the simulation scenario where six WLAN cells are covered by a UMTS cellular network. The scenario involves an ambulance or an MT traveling from point $\mathrm{A}$ to point $\mathrm{B}$ crossing WLAN_1, WLAN_2, WLAN_3 and WLAN_4. The actual traveling distance $\bar{l}$ within WLAN_1, WLAN_2, WLAN_3 and WLAN_4 is $71.4 \mathrm{~m}, 43.6 \mathrm{~m}, 34.1 \mathrm{~m}$ and $19.9 \mathrm{~m}$, respectively.

The performance of THODM algorithm is compared with the RSS threshold based handover (RSS) algorithm, Cellular based scheme and ideal solution. The RSS threshold based handover algorithm triggers handover to WLAN whenever $R S S_{W L A N}$ is greater than $R S S_{t h}$. Cellular based scheme always connect to the UMTS network. It represents the existing handover algorithms which set the speed threshold for WLAN and only select the WWAN at high mobility. Ideal solution is a theoretical result of MT connection to WLAN and UMTS network while traveling from point A to point B without any unnecessary handover.

It is assumed network providers reserve certain network channels at each UMTS base station and WLAN access point for telemedicine purpose [11]. These reserved channels have average throughput of 1 Mbps [24] and 6 Mbps [19] for UMTS and WLAN, respectively. Therefore, telemedicine user does not have to worry about the network channel availability. The average transmission cost of WLAN and UMTS is 1 and 5 units cost per $\mathrm{Mb}$, respectively [19].

The experiment is simulated for 100 loops. The starting point $\mathrm{A}$ is set randomly within the range of $\delta$ (as shown in Fig. 4) so that the ambulance or MT has different starting point A in every loop. The experiment parameter settings are shown in Table III. 


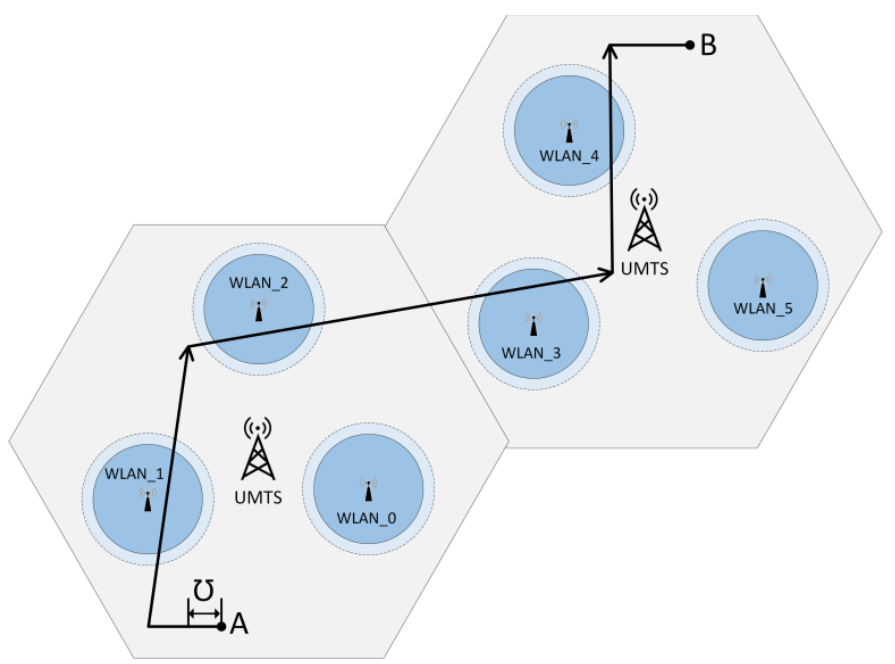

Fig. 4. Simulation scenario in heterogeneous wireless networks (MT travels from point A to point B)

TABLE III. SIMULATION METRICS

\begin{tabular}{ll}
\hline Parameter & Value \\
\hline$D R_{R E Q}(\mathrm{kbps})$ & $\begin{array}{l}\text { Video + ECG + Voice + Heart } \\
\text { rate } \approx 800 \mathrm{kbps}\end{array}$ \\
\hline$P_{T X}$ & $100 \mathrm{~mW}[20]$ \\
$n$ & $3.5[36]$ \\
\hline$\varepsilon$ & $4.3 \mathrm{~dB}[36]$ \\
\hline RSS at boundary, RSS $_{\text {boundary }}$ & $-76.61 \mathrm{dBm}$ \\
\hline RSS threshold, RSS & $-75.16 \mathrm{dBm}$ \\
\hline WLAN data rate $(\mathrm{Mbps})$ & $6[40]$ \\
\hline UMTS data rate $(\mathrm{Mbps})$ & $1[24]$ \\
\hline WLAN cost per Mb (unit) & $1[19]$ \\
\hline Cellular cost per Mb (unit) & $5[19]$ \\
\hline$R$ (m) & 55 \\
\hline$r(\mathrm{~m})$ & $50[18]$ \\
$v$ & 50 to $120 \mathrm{~km} / \mathrm{h}$ \\
\hline Monitoring time interval (s) & $1 \mathrm{~m} / v$ \\
\hline$T_{W L A N \_t h}(\mathrm{~s})$ & $2[18]$ \\
\hline$\sigma(\mathrm{m})$ & Random [0 5] \\
\hline$\varphi$ & 0.2 \\
\hline
\end{tabular}

\section{A. Unnecessary Handover}

Unnecessary handover is defined as a handover that does not benefit the user. It occurs when user failed to establish connection with targeted network due to an abnormal call release or inappropriate handover and the reestablishment of a connection with previous network is required. The number of unnecessary handover (NUHO) can be determined by

$$
\mathrm{NUHO}_{a}=\mathrm{NHO}_{a}-\mathrm{NHO}_{\text {ideal }} \quad, a \in\{\mathrm{THODM}, \mathrm{RSS}\} \quad \text { (23) }
$$

where $\mathrm{NHO}_{\text {ideal }}$ is total number of handover achieved by ideal solution. In ideal solution, any handovers to WLAN_4 will be considered as unnecessary at the speed of $35 \mathrm{~km} / \mathrm{h}$ and above because $T_{W L A N}$ in WLAN_4 $\left(T_{\text {WLAN_4 }}\right)$ is less than $T_{W L A N \_t h}$. Similar to WLAN_3, WLAN_2 and WLAN_1, an unnecessary handover occurs when the velocity of MT is higher than 61, 78 and $128 \mathrm{~km} / \mathrm{h}$, respectively. Fig. 5 and Fig. 6 show the total number of handover and unnecessary handover occurred in THODM, RSS and ideal solution schemes.

The result in Fig. 6 shows that THODM algorithm has less number of unnecessary compared to RSS scheme. This is contribution of dwelling time prediction process which rejects all the WLAN cells that estimated $T_{\text {WLAN }}$ is less than $T_{\text {WLAN_th }}$ even though these WLAN cells have better network quality than UMTS.

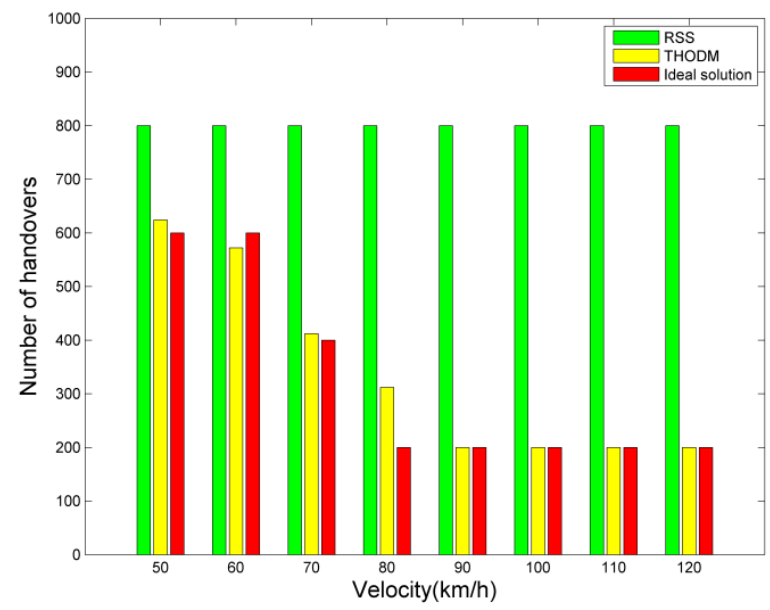

Fig. 5. Total number of handovers

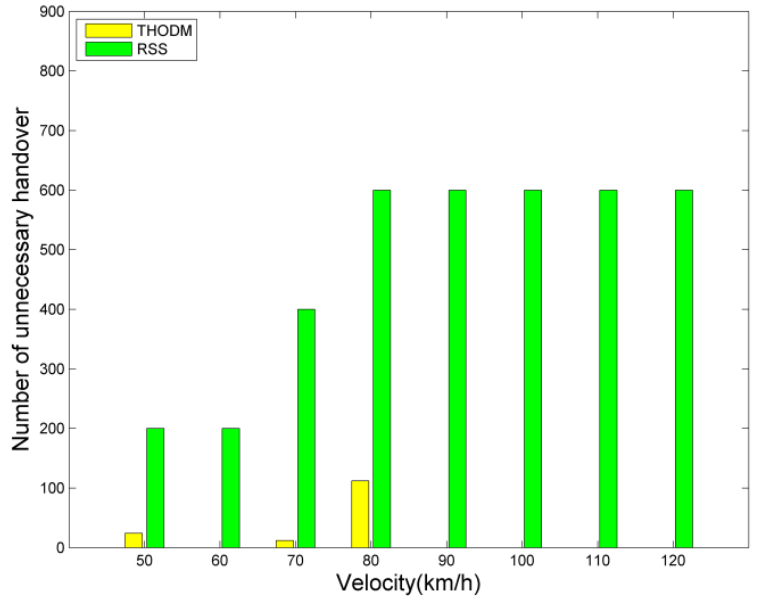

Fig. 6. Number of unnecessary handovers

\section{B. Throughput}

The total throughput achieved by MT is dependent on the connection time with UMTS and WLAN, respectively. However, it is affected by the number of unnecessary handovers. The total throughput $\left(T_{\text {Throughput }}\right)$ achieved by MT can be determined by [24] 


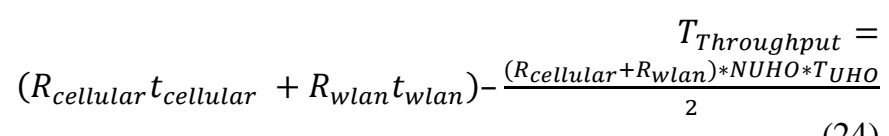

where $T_{U H O}$ is time consumed by each unnecessary handover ( 2 seconds) [18], $R_{\text {cellular }}$ and $R_{\text {wlan }}$ represent average data rate of UMTS and WLAN, $t_{\text {cellular }}$ and $t_{\text {wlan }}$ denotes total time connected to UMTS and WLAN, respectively.

Fig. 7 shows the average throughput achieved by MT based on different approaches (Cellular, RSS, THODM, and ideal solution) in single loop (from point A to point B) at speed of $50 \mathrm{~km} / \mathrm{h}$ to $120 \mathrm{~km} / \mathrm{h}$. The average throughput decreases when the MT's velocity increases. This is because MT takes less time to travel from point A to B. It can be seen that the total throughput obtained by the proposed THODM algorithm is higher than RSS and Cellular schemes. Furthermore, the throughput achieved by the THODM algorithm is proximate to ideal solution. by

The percentage of throughput gain $(G)$ can be determined

$$
G=\frac{T P_{T H O D M}-T P_{x}}{T P_{x}} \times 100 \%, \quad x \in\{\text { Cellular, RSS }\}
$$

where $T P_{\text {THODM }}$ is total throughput achieved by THODM and $T P_{x}$ represents total throughput of RSS or Cellular based scheme. As depicted in Fig. 8, the throughput of THODM is up to $75 \%$ and $205 \%$ higher than Cellular and RSS schemes, respectively.

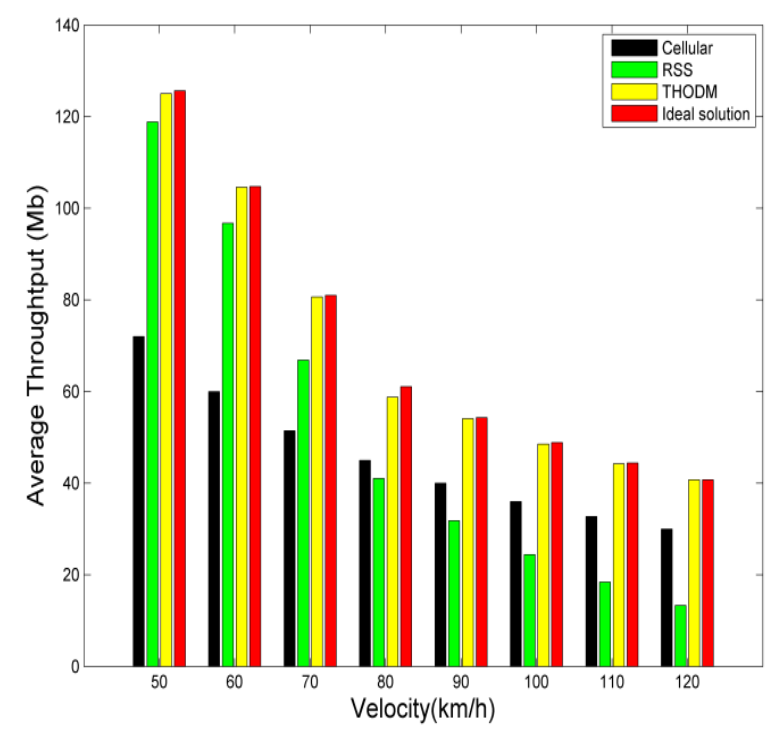

Fig. 7. Average throughput achieved by MT in single loop at the speed of $50 \mathrm{~km} / \mathrm{h}$ to $120 \mathrm{~km} / \mathrm{h}$.

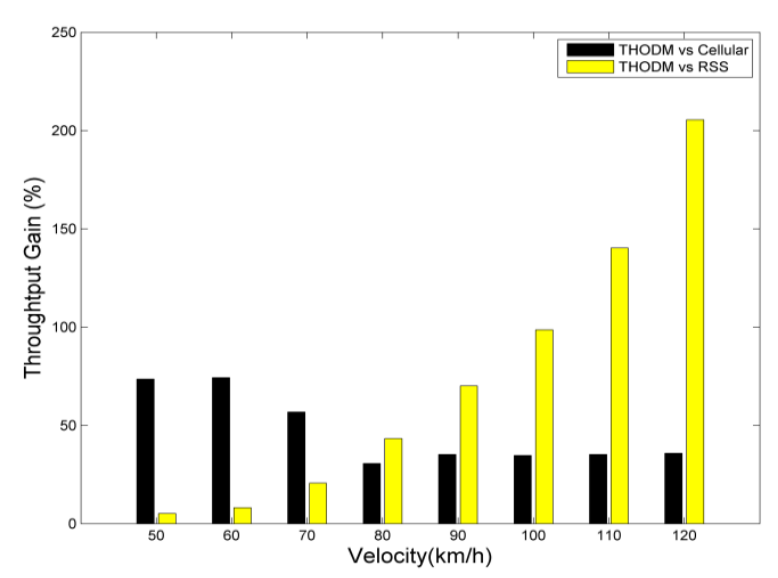

Fig. 8. Percentage of throughput gain

\section{Transmission Cost}

Assumed the transmission cost of UMTS is five times higher than WLAN [19]. The transmission cost per Mb (C) can be calculated by

$$
C=\frac{R_{\text {cellular }} t_{\text {cellular }} C_{\text {cellular }}+R_{\text {wlan }} t_{w l a n} C_{\text {wlan }}+N_{H O} C_{H O}}{T_{\text {Throughput }}}
$$

where $N_{H O}$ is number of handover, $C_{H O}$ represents handover cost (predefined $C_{H O}=3$ units), $C_{\text {wlan }}$ and $C_{\text {cellular }}$ denote average cost per $\mathrm{Mb}$ offered by WLAN and cellular network. As can be seen in Fig. 9, THODM algorithm has the lowest average cost per $\mathrm{Mb}$ compared to RSS and Cellular schemes. At speed of $120 \mathrm{~km} / \mathrm{h}$, the average cost of THODM is $24 \%$ and $69.2 \%$ lower than Cellular and RSS schemes, respectively.

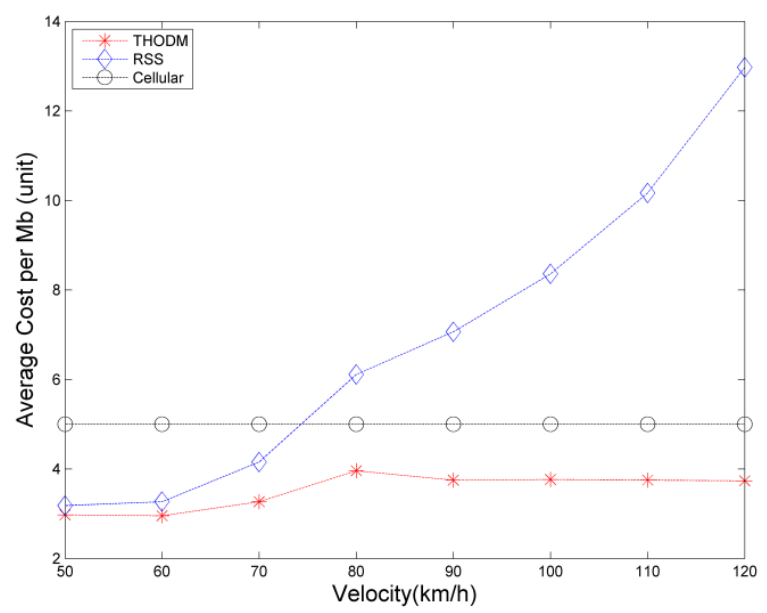

Fig. 9. Average cost per $\mathrm{Mb}$ 


\section{CONCLUSION}

This paper presented a THODM algorithm to support telemedicine service in high speed heterogeneous environment. The proposed algorithm resolves the problems such as limited coverage and mobility issue faced by the existing mobile telemedicine system by selecting the best network according to the services requirement. The dwelling time prediction process in THODM algorithm has successfully reduced the number of unnecessary handovers while optimizing the usage of WLAN in high speed environment. The simulation results show that the proposed algorithm has higher throughput and more cost effective than RSS and Cellular schemes. The proposed THODM algorithm is suitable for ambulance based mobile telemedicine system.

The limitation of this work is that we assume the MT moves at constant speed when crossing the WLAN coverage. For future work, we will enhance the dwelling time prediction method in THODM algorithm so that it can accurately estimate the MT's dwelling time in WLAN coverage even though MT moves in dynamic speed.

\section{REFERENCES}

[1] J. C. Tejero-Calado, C. Lopez-Casado, A. Bernal-Martin, M. A. LopezGomez, M. A. Romero-Romero, G. Quesada, et al., "IEEE 802.11 ECG monitoring system," in Engineering in Medicine and Biology Society, 2005. IEEE-EMBS 2005. 27th Annual International Conference of the, 2005, pp. 7139-7142.

[2] A. Moein and M. Pouladian, "WIH-Based IEEE 802.11 ECG Monitoring Implementation," in Engineering in Medicine and Biology Society, 2007. EMBS 2007. 29th Annual International Conference of the IEEE, 2007, pp. 3677-3680.

[3] P. A. Sakthi and R. Sukanesh, "A reliable and fast routing data transmission protocol for Wi-Fi based real-time patient monitoring system," in Electronics and Communication Systems (ICECS), 2014 International Conference on, 2014, pp. 1-5.

[4] K. Cai and X. Liang, "Development of WI-FI Based Telecardiology Monitoring System," in Intelligent Systems and Applications (ISA), 2010 2nd International Workshop on, 2010, pp. 1-4.

[5] S. Mitra, M. Mitra, and B. B. Chaudhuri, "Rural cardiac healthcare system-A scheme for developing countries," in TENCON 2008 - 2008 IEEE Region 10 Conference, 2008, pp. 1-5.

[6] M. Abo-Zahhad, S. M. Ahmed, and O. Elnahas, "A wireless emergency telemedicine system for patients monitoring and diagnosis," Int J Telemed Appl, vol. 2014, p. 380787, 2014.

[7] H. Anpeng, C. Chao, B. Kaigui, D. Xiaohui, C. Min, G. Hongqiao, et al., "WE-CARE: An Intelligent Mobile Telecardiology System to Enable mHealth Applications," Biomedical and Health Informatics, IEEE Journal of, vol. 18, pp. 693-702, 2014.

[8] H. Mateev, I. Simova, T. Katova, and N. Dimitrov, "Clinical Evaluation of a Mobile Heart Rhythm Telemonitoring System," ISRN Cardiology, vol. 2012, p. 8, 2012

[9] I. Chorbev and M. Mihajlov, "Wireless telemedicine services as part of an integrated system for e-medicine," in Electrotechnical Conference, 2008. MELECON 2008. The 14th IEEE Mediterranean, 2008, pp. 264269.

[10] D. Niyato, E. Hossain, and J. Diamond, "IEEE 802.16/WiMAX-based broadband wireless access and its application for telemedicine/e-health services," Wireless Communications, IEEE, vol. 14, pp. 72-83, 2007.

[11] D. Niyato, E. Hossain, and S. Camorlinga, "Remote patient monitoring service using heterogeneous wireless access networks: architecture and optimization," Selected Areas in Communications, IEEE Journal on, vol. 27, pp. 412-423, 2009.

[12] D. Pareit, B. Lannoo, I. Moerman, and P. Demeester, "The History of WiMAX: A Complete Survey of the Evolution in Certification and
Standardization for IEEE 802.16 and WiMAX," Communications Surveys \& Tutorials, IEEE, vol. 14, pp. 1183-1211, 2012.

[13] I. F. Akyildiz, J. McNair, J. S. M. Ho, H. Uzunalioglu, and W. Wenye, "Mobility management in next-generation wireless systems," Proceedings of the IEEE, vol. 87, pp. 1347-1384, 1999.

[14] M. Aguado, J. Astorga, J. Matias, and M. Huarte, "The MIH (Media Independent Handover) Contribution to Mobility Management in a Heterogeneous Railway Communication Context: A IEEE802.11/802.16 Case Study," in Communication Technologies for Vehicles. vol. 6596, T. Strang, A. Festag, A. Vinel, R. Mehmood, C. Rico Garcia, and M. Röckl, Eds., ed: Springer Berlin Heidelberg, 2011, pp. 69-82.

[15] S. Mohanty and I. F. Akyildiz, "A Cross-Layer (Layer $2+3$ ) Handoff Management Protocol for Next-Generation Wireless Systems," Mobile Computing, IEEE Transactions on, vol. 5, pp. 1347-1360, 2006.

[16] S. Mohanty, "A new architecture for 3G and WLAN integration and inter-system handover management," Wireless Networks, vol. 12, pp. 733-745, 2006/12/01 2006.

[17] R. Hussain, S. Malik, S. Abrar, R. Riaz, H. Ahmed, and S. Khan, "Vertical Handover Necessity Estimation Based on a New Dwell Time Prediction Model for Minimizing Unnecessary Handovers to a WLAN Cell," Wireless Personal Communications, vol. 71, pp. 1217-1230, 2013/07/01 2013.

[18] Y. Xiaohuan, N. Mani, and Y. A. Sekercioglu, "A Traveling Distance Prediction Based Method to Minimize Unnecessary Handovers from Cellular Networks to WLANs," Communications Letters, IEEE, vol. 12, pp. 14-16, 2008.

[19] K. Hong, S. Lee, L. Kim, and P. Song, "Cost-based vertical handover decision algorithm for WWAN/WLAN integrated networks," EURASIP J. Wirel. Commun. Netw., vol. 2009, pp. 1-11, 2009.

[20] Y. Kemeng, I. Gondal, and Q. Bin, "Multi-Dimensional Adaptive SINR Based Vertical Handoff for Heterogeneous Wireless Networks," Communications Letters, IEEE, vol. 12, pp. 438-440, 2008.

[21] E. M. Malathy and V. Muthuswamy, "Knapsack - TOPSIS Technique for Vertical Handover in Heterogeneous Wireless Network," PLoS ONE, vol. 10, p. e0134232, 2015.

[22] R. Tawil, G. Pujolle, and O. Salazar, "A Vertical Handoff Decision Scheme in Heterogeneous Wireless Systems," in Vehicular Technology Conference, 2008. VTC Spring 2008. IEEE, 2008, pp. 2626-2630.

[23] S.-m. Liu, S. Pan, Z.-k. Mi, Q.-m. Meng, and M.-h. Xu, "A Simple Additive Weighting Vertical Handoff Algorithm Based on SINR and AHP for Heterogeneous Wireless Networks," in Intelligent Computation Technology and Automation (ICICTA), 2010 International Conference on, 2010, pp. 347-350.

[24] A. Singhrova and N. Prakash, "Vertical handoff decision algorithm for improved quality of service in heterogeneous wireless networks," Communications, IET, vol. 6, pp. 211-223, 2012.

[25] F. Kaleem, A. Mehbodniya, K. K. Yen, and F. Adachi, "A Fuzzy Preprocessing Module for Optimizing the Access Network Selection in Wireless Networks," Advances in Fuzzy Systems, vol. 2013, p. 9, 2013.

[26] N. Nasser, S. Guizani, and E. Al-Masri, "Middleware Vertical Handoff Manager: A Neural Network-Based Solution," in Communications, 2007. ICC '07. IEEE International Conference on, 2007, pp. 5671-5676.

[27] W. Song and W. Zhuang, "Introduction on Cellular/WLAN Interworking," in Interworking of Wireless LANs and Cellular Networks, ed: Springer New York, 2012, pp. 1-10.

[28] M. Khan and K. Han, "An Optimized Network Selection and Handover Triggering Scheme for Heterogeneous Self-Organized Wireless Networks," Mathematical Problems in Engineering, vol. 2014, p. 11, 2014.

[29] H. T. Yew, E. Supriyanto, M. Haikal Satria, and Y. W. Hau, "Usercentric based vertical handover decision algorithm for telecardiology application in heterogeneous networks," Jurnal Teknologi, vol. 77, pp. 79-83, 2015.

[30] T. Janevski and K. Jakimoski, "Mobility sensitive algorithm for vertical handovers from WiMAX to WLAN," in Telecommunications Forum (TELFOR), 2012 20th, 2012, pp. 91-94.

[31] H. T. Yew, Y. Aditya, H. Satrial, E. Supriyanto, and Y. W. Hau, "Telecardiology system for fourth generation heterogeneous wireless 
networks," ARPN Journal of Engineering and Applied Sciences, vol. 10, pp. 600-607, 2015.

[32] J. R. Gallego, A. Hernandez-Solana, M. Canales, J. Lafuente, A. Valdovinos, and J. Fernandez-Navajas, "Performance analysis of multiplexed medical data transmission for mobile emergency care over the UMTS channel," IEEE Trans Inf Technol Biomed, vol. 9, pp. 13-22, Mar 2005.

[33] C. Yuechun and A. Ganz, "A mobile teletrauma system using 3G networks," Information Technology in Biomedicine, IEEE Transactions on, vol. 8, pp. 456-462, 2004.

[34] H. T. Yew, E. Supriyanto, M. H. Satria, and Y. W. Hau, "Adaptive network selection mechanism for telecardiology system in developing countries," in 2016 IEEE-EMBS International Conference on Biomedical and Health Informatics (BHI), 2016, pp. 94-97.

[35] P. Santi, "Modeling Next Generation Wireless Networks," in Mobility Models for Next Generation Wireless Networks, ed: John Wiley \& Sons, Ltd, 2012, pp. 19-32.
[36] Y. Xiaohuan, Y. A. Sekercioglu, and N. Mani, "A method for minimizing unnecessary handovers in heterogeneous wireless networks," in World of Wireless, Mobile and Multimedia Networks, 2008. WoWMoM 2008. 2008 International Symposium on a, 2008, pp. $1-5$.

[37] B. Singh, "An improved handover algorithm based on signal strength plus distance for interoperability in mobile cellular networks," Wireless Personal Communications, vol. 43, pp. 879-887, 2007.

[38] Y. Yang, P. Fan, and Y. Huang, "Doppler frequency offsets estimation and diversity reception scheme of high speed railway with multiple antennas on separated carriages," in Wireless Communications \& Signal Processing (WCSP), 2012 International Conference on, 2012, pp. 1-6.

[39] E. A. Feukeu, K. Djouani, and A. Kurien, "Compensating the effect of Doppler shift in a vehicular network," in AFRICON, 2013, 2013, pp. 17.

[40] Z. Yan, N. Ansari, and H. Tsunoda, "Wireless telemedicine services over integrated IEEE 802.11/WLAN and IEEE 802.16/WiMAX networks," Wireless Communications, IEEE, vol. 17, pp. 30-36, 2010. 